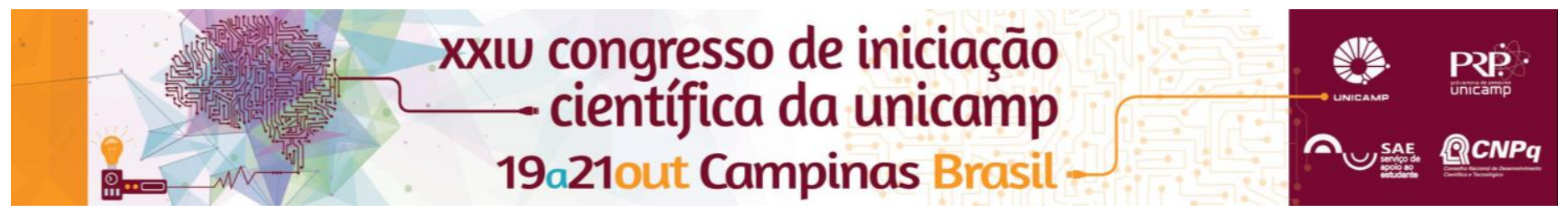

\title{
Ultrasound-assisted extraction of antioxidants present in roasted coffee beans (Coffea Arábica L.)
}

\section{Gustavo Henrique Goebel*, Mauricio Ariel Rostagno, Julian Martinez, Ana Paula F. Machado, Maria Del Pilar G. Mendoza.}

\begin{abstract}
The use of ultrasound as an assistant in the compounds extraction has been highlighted by the high efficiency. The focus of this work were the phenolic compounds of great interest today by its antioxidant activity, and which are able to be found worldwide through the coffee in the form of phenolic acids. Various conditions of temperature and frequency were evaluated to find the parameters that increase higher efficiency at the end of the extraction.
\end{abstract}

Key words: Extraction, Ultrasound, Antioxidants.

\section{Introduction}

Coffee, in addition to caffeine, has many other bioactive compounds such as phenolic compounds represented by chlorogenic acids and their various isomers. The antioxidant activity of the phenolic compounds is due primarily to their reducing properties and chemical structure, which act in the oxidative process steps, forming relatively stable intermediates and therefore provide benefits to human health.

To be able to extract these compounds one of the techniques that have been studied in various foods is ultrasound. The ultrasound effects arise from cavitation phenomena caused by irradiation of ultrasonic waves through the medium. The ultrasound advantages are extensive and overcome the deficiencies of conventional techniques on the consumption of solvent and energy, allowing a shorter treatment time at a low cost implementation.

The aim of this study was to perform extractions with the use of ultrasound at different frequencies and temperatures, to evaluate which were the most efficient conditions in the extraction of antioxidant compounds of roasted coffee beans. For the determination of total phenolic content was used Folin -Ciocalteu method and for the antioxidant activity was used the DPPH scavenging method and Ferric Reducing Antioxidant Power (FRAP). In this step, the solvent used was ethanol $50 \%$ and the extraction time was set at 15 minutes. The roasted coffee beans of two marks were ground for the extractions.

\section{Results and Discussion}

The ultrasound bath used has the 37 and $80 \mathrm{kHz}$ frequency and it can be combined with the sweep and degas modes, which cause the best effect of ultrasound scanning and pulses. At the end, six different frequency conditions were tested with four temperatures, 40, 50, 60 and $70^{\circ} \mathrm{C}$.

The total phenolic analysis presented as better condition the frequency of $37 \mathrm{kHz}$ with a temperature of $60{ }^{\circ} \mathrm{C}$, reaching a maximum of $37.12 \mathrm{mg}$ of Gallic acid per gram of dry sample, Image 1. This result was also repeated in DPPH analysis. In contrast, the analysis of FRAP reagent, the best condition was found at $80 \mathrm{kHz}$ at $70^{\circ} \mathrm{C}$. It was expected that higher temperatures favor the DOI: 10.19146/pibic-2016-50813 extraction, however, the best result was obtained with a temperature of $60^{\circ} \mathrm{C}$

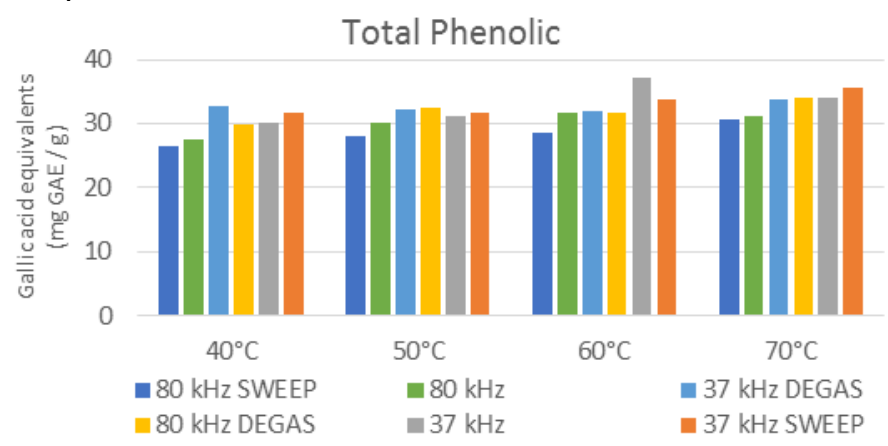

Image 1. Content of total phenolic between conditions

\section{Conclusions}

From the results shown by the analysis, among the ultrasound conditions which the samples were submitted, one that obtained the highest extraction efficiency was the frequency of $37 \mathrm{kHz}$ combined with a temperature of $60^{\circ} \mathrm{C}$, for indeed, the extracts of this condition were those with the highest content of antioxidants and phenolic compounds.

\section{Acknowledgement}

I would like to thanks my family, my guiding Mauricio and my laboratory colleagues that supported me and contributed to the completion of this work. The CNPq for the scholarship. Unicamp, DEA and LAPEA for the institutional support.

ILLY, A.; VIANI, R.; Espresso Coffee: the Science of Quality; 2nd ed., Elsevier Academic Press: London, 2005

PASQUEL REÁTEGUI. et al. Extraction of antioxidant compounds from blackberry (Rubus sp.) bagasse using supercritical $\mathrm{CO} 2$ assisted by ultrasound. Journal of Supercritical Fluids 94 (2014) 223-233.

ROSTAGNO, M. A.; PRADO, J. M. (Ed). Natural Product Extraction Principles and Applications. RSC Publishing. Cambridge, UK. ISBN: 978-1-84973-606-0., 2013 .

PREEDY, V.R., ed. Coffee in Health and Disease Prevention. Academic Press, 2014 . 